\title{
Mitochondrial fission is an upstream and required event for bax foci formation in response to nitric oxide in cortical neurons
}

\author{
H Yuan ${ }^{1,2,4}$, AA Gerencser ${ }^{1,4}$, G Liot ${ }^{1}$, SA Lipton ${ }^{2}$, M Ellisman ${ }^{3}$, GA Perkins ${ }^{3}$ and E Bossy-Wetzel ${ }^{\star 1,2}$
}

\begin{abstract}
Mitochondrial dysfunction is an underpinning event in many neurodegenerative disorders. Less clear, however, is how mitochondria become injured during neuronal demise. Nitric oxide (NO) evokes rapid mitochondrial fission in cortical neurons. Interestingly, proapoptotic Bax relocates from the cytoplasm into large foci on mitochondrial scission sites in response to nitrosative stress. Antiapoptotic Bcl-xL does not prevent mitochondrial fission despite its ability to block Bax puncta formation on mitochondria and to mitigate neuronal cell death. Mitofusin 1 (Mfn1) or dominant-negative dynamin-related protein $1^{\mathrm{K} 38 \mathrm{~A}}$ $\left(\right.$ Drp $\left.^{\mathbf{k} 38 \mathrm{~A}}\right)$ inhibits mitochondrial fission and Bax accumulation on mitochondria induced by exposure to an NO donor. Although NO is known to cause a bioenergetic crisis, lowering ATP by glycolytic or mitochondrial inhibitors neither induces mitochondrial fission nor Bax foci formation on mitochondria. Taken together, these data indicate that the mitochondrial fission machinery acts upstream of the Bcl-2 family of proteins in neurons challenged with nitrosative stress.
\end{abstract}

Cell Death and Differentiation (2007) 14, 462-471. doi:10.1038/sj.cdd.4402046; published online 20 October 2006

Nitric oxide (NO) plays a pivotal role in normal cell physiology. In brown fat tissue NO regulates mitochondrial biogenesis. ${ }^{1}$ In the central nervous system, NO functions as an important neurotransmitter, but when overproduced by excessive glutamate receptor activation, $\mathrm{NO}$ reacts with superoxide anion $\left(\mathrm{O}_{2}^{\bullet-}\right)$ to form peroxynitrite $\left(\mathrm{ONOO}^{-}\right)$, a highly reactive, neurotoxic molecule. ${ }^{2,3}$ NO-mediated neuronal injury is implicated in several neurodegenerative disorders, including stroke, Parkinson's disease, Alzheimer's disease, Huntington's disease, and ALS. $^{4} \mathrm{NO}^{-\mathrm{ONOO}^{-}}$inhibits mitochondrial respiration $^{5-7}$ and $\mathrm{ONOO}^{-}$liberates $\mathrm{Zn}^{2+}$ from endogenous stores, which in turn triggers further mitochondrial injury. ${ }^{8}$ Besides these properties, $\mathrm{NO} / \mathrm{ONOO}^{-}$activates signaltransduction pathways of mitogen-activated kinases, which can participate in neuronal demise. ${ }^{9}$ The mechanism underlying nitrosative stress-mediated neuronal cell death is not fully understood, but mitochondrial injury appears to be central. ${ }^{6}$ Thus a better understanding of the mechanism underlying mitochondrial injury during neurodegeneration is required.

Mitochondria are dynamic organelles, undergoing frequent fission and fusion. These opposing processes are choreographed by a conserved group of large GTPases. Their balanced activities dictate mitochondrial morphology, size, and number. Dynamin-related protein 1 (Drp1), which is found in the cytoplasm and on mitochondria, has emerged as key mitochondrial fission factor. ${ }^{10,11}$ Upon activation by an unknown mechanism, Drp1 accumulates at mitochondria and preferentially localizes to future fission sites. ${ }^{12}$ Conversely, mitochondrial fusion is regulated by large GTPases, such as Mitofusin1 and 2 (Mfn1 and 2) and optic atrophy1 (OPA1). ${ }^{13}$ Mitochondrial fusion requires an intact mitochondrial membrane potential $\left(\Delta \Psi_{\mathrm{m}}\right) \cdot{ }^{14}$ Loss of either Mfn1, 2, or OPA1 results in mitochondrial fission and cellular dysfunction. ${ }^{15,16}$

Mitochondrial division is a normal physiological process; however, extensive mitochondrial division or fission can occur under patho-physiological conditions. ${ }^{13,17}$ Evidence has emerged indicating that mitochondrial fission plays an active part in apoptotic cell death. ${ }^{12,18}$

We have previously shown that NO-induced mitochondrial fission plays a causal role in neuronal demise in cultured neurons. ${ }^{17}$ Although the NO donor S-nitrosocysteine $(\mathrm{SNOC})^{19}$ triggers mitochondrial fission and cell death in cortical neurons, ${ }^{17}$ it is still unclear how mitochondrial fission contributes to NO-mediated neuronal cell death. To address this question we set out to test the effects of NO donors on mitochondrial fission and its relationship to neuronal cell death events in neurons of primary cortical cultures. Here, we show that NO triggers rapid mitochondrial fission and formation of

\footnotetext{
${ }^{1}$ Apoptosis and Cell Death Program, Burnham Institute for Medical Research, 10901 N. Torrey Pines Rd., La Jolla, CA 92037, USA; ${ }^{2}$ Center for Neuroscience and Aging, Burnham Institute for Medical Research, 10901 N. Torrey Pines Rd., La Jolla, CA 92037, USA and ${ }^{3}$ National Center for Microscopy and Imaging Research, School of Medicine, University of California, San Diego, La Jolla, CA 92093, USA

*Corresponding author: E Bossy-Wetzel, Apoptosis and Cell Death Program, Burnham Institute for Medical Research, 10901 North Torrey Pines Rd., La Jolla, CA 92037, USA. Tel: 858-746-3100; Ext. 5297; Fax: 858-646-3196; E-mail: ebossy-wetzel@ burnham.org

${ }^{4}$ These authors contributed equally to this work

Keywords: nitric oxide; Bcl-2 family proteins; Drp1; Mfn; neurodegeneration

Abbreviations: Mfn1, mitofusin 1; Drp1, dynamin-related protein 1; OPA1, optic atrophy 1; SNOC, S-nitrosocysteine; FCCP, carbonyl cyanide 4-(trifluoromethoxy) phenylhydrazone; TEM, transmission EM; SNAP, nitroso- $\mathrm{N}$-acetylpenicillamine; NAC, $\mathrm{N}$-acetyl-cysteine; GSH, reduced glutathione; Hb, hemoglobin; 2DG 2-deoxyglucose; GAPDH, glyceraldehydes-3-phospate dehydrogenase; AICAR, 5-aminoimidazole-4-casrboxaminde ribonuceloside; HBSS, HEPES-buffered salt solution; $\Delta \Psi_{\mathrm{m}}$, mitochondrial membrane potential

Received 13.2.06; revised 04.9.06; accepted 04.9.06; Edited by M Piacentini; published online 20.10.06
} 
Bax aggregates (foci) on mitochondria through nitrosative stress. Preventing fission by modulating the activity of dynamin-related GTPases like Mfn1 and Drp1 ${ }^{\mathrm{K} 38 \mathrm{~A}}$ inhibits Bax foci on mitochondria. Bcl-xL did not prevent fission, but prevents Bax accumulation on mitochondria and alleviates neuronal cell death. Our results suggest that fission acts upstream of $\mathrm{Bcl}-2$ related proteins in neurons undergoing cell death by nitrosative stress.

\section{Results}

NO donors induce mitochondrial fission. To determine the effect of NO on mitochondrial dynamics, we transiently transfected primary mixed cortical cultures with a vector encoding red fluorescent protein, DsRed2, fused to the mitochondrial targeting sequence of human cytochrome $c$ oxidase subunit VIII. DsRed2-Mito selectively traces the mitochondrial matrix. Our neuronal cultures are of mixed type, containing neurons, astrocytes, and microglia, therefore mimic an in vivo environment. SNOC, a NO donor, is commonly being used in studies of NO-mediated neurotoxicity in cultured neurons. $7,17,19$ The reaction of NO depends upon the redox state of the individual neuron. ${ }^{20}$ Here we monitored SNOC-induced changes in mitochondrial morphology by 3D fluorescence microscopy. Neurons were identified by their pyramidal cell bodies and long processes. In healthy neurons, mitochondria in neuronal processes form elongated tubules of varying length (Figure 1a). Following exposure to $200 \mu \mathrm{M}$ SNOC, a concentration often resulting in neuronal cell death, ${ }^{17}$ mitochondrial filaments underwent profound fission, forming numerous small rounded and isolated organelles (Figure 1b). Once started, mitochondrial fission occurred within 10-20 min and affected all mitochondria within a neuron.

To confirm our results with SNOC, we tested the effects of other NO donors including Spermine NONOate and nitroso$\mathrm{N}$-acetylpenicillamine (SNAP) on mitochondrial morphology. Spermine NONOate releases free $\mathrm{NO}$ into an aqueous solution and has a half-life of $\mathbf{2 3 0} \mathrm{min}$, whereas SNAP, which donates NO to other thiols has a half-life of $10 \mathrm{~h}$. Similar to SNOC, Spermine NONOate induces concentration-dependent mitochondrial fission at $4 \mathrm{~h}$ (Figure $1 \mathrm{c}$ ). In addition, SNAP evoked dose-dependent mitochondrial fission, however, only at $12 \mathrm{~h}$ after exposure. The discrepacy in timing could be due to differences in release rates, stability, and the type of NO species formed among the different NO donors.

We recently demonstrated that SNOC exposure increases free radical production in neurons. ${ }^{8,17}$ We therefore tested whether scavenging nitrosative and oxidative stress with $\mathrm{N}$ acetyl-cysteine (NAC) $(150 \mu \mathrm{M})$, reduced glutathione (GSH) $(200 \mu \mathrm{M})$, or hemoglobin $(\mathrm{Hb}$; oxidized) $(75 \mu \mathrm{M})$, would prevent SNOC-induced mitochondrial fission. All three agents significantly abolished SNOC-evoked mitochondrial fission (Figure 1e and $\mathrm{f}$ ). These results suggest that SNOC triggers mitochondrial fission through nitrosative stress.

Nitrosative stress induces ultrastructural changes of mitochondria. To confirm our results using light microscopy we carried out transmission electron microscopy (TEM). In
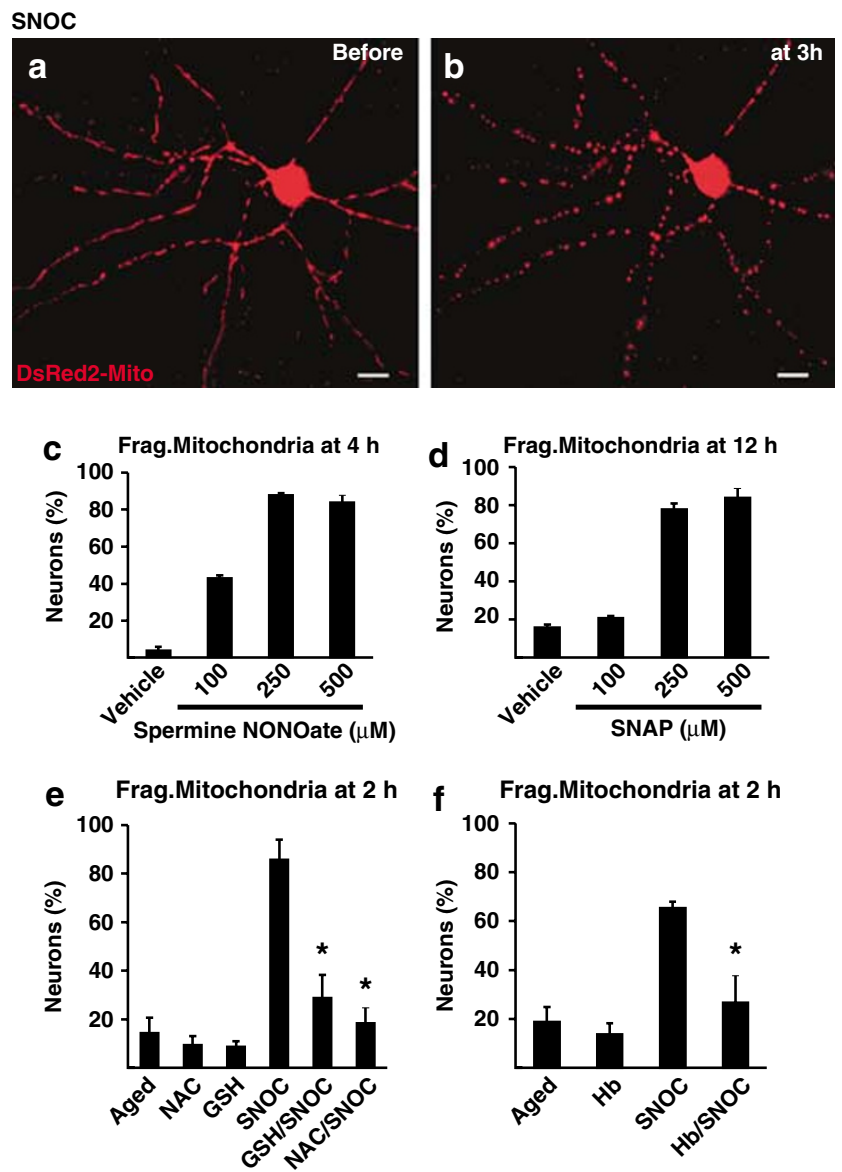

Figure 1 NO donors induce mitochondrial fission in cortical neurons. (a) Fluorescence images of a DsRed2-Mito transfected cortical neuron. Before SNOC exposure mitochondria show elongated and tubular morphology. (b) The same neuron was exposed to $200 \mu \mathrm{M}$ SNOC. At $3 \mathrm{~h}$ mitochondria are present as small spheres. Scale bar, $10 \mu \mathrm{m}$. (c) Spermine NONOate induces concentrationdependent mitochondrial fission at $4 \mathrm{~h}$. (d) SNAP induces concentration-dependent mitochondrial fission at $12 \mathrm{~h}$. (e) NAC $(150 \mu \mathrm{M})$ and GSH $(200 \mu \mathrm{M})$ decreased mitochondrial fission induced by SNOC $(175 \mu \mathrm{M})$ at $2 \mathrm{~h}$. Neurons with $90 \%$ round and small mitochondria were scored as ones with fragmented mitochondria. (f) $\mathrm{Hb}$ $(75 \mu \mathrm{M})$ mitigates mitochondrial fission by SNOC $(175 \mu \mathrm{M})$ at $2 \mathrm{~h}$. Data are mean \pm S.E.M. and representative of three independent experiments $\left({ }^{\star} P<0.05\right.$ compared to SNOC samples)

control cultures, mitochondria close to the nucleus (Figure $2 \mathrm{a}$ ) and in neuronal processes (Figure $2 \mathrm{~b}$ ) revealed typical elongated filamentous morphology when evaluated in EM micrographs. In contrast, mitochondria in SNOC-treated cortical cultures were shorter in length and had a more rounded shape close to the nucleus and in neuronal projections (Figure $2 c$ and $d$ ). This was without major loss of matrix electron density or cristae structure (Figure 2d). There was no clustering of mitochondria in the perinuclear region after SNOC treatment (Figure $2 \mathrm{c}$ and d). Swollen mitochondria with clear loss of matrix electron density were only seen in more severely injured dendrites (Figure 2e). However, it is important to note that the occurrence of swollen mitochondria was a rare event, therefore most likely of secondary nature.

The number of mitochondria and volume density were quantified by stereology. Stereological measurements 


\section{Control}

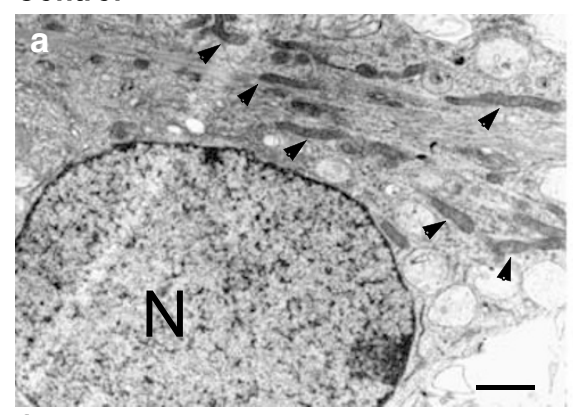

\section{Control}

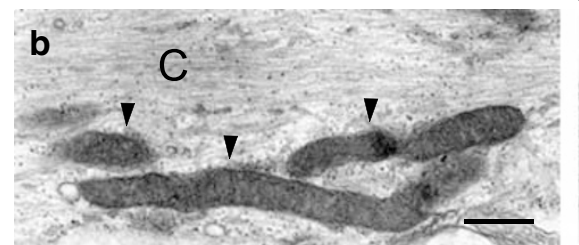

f

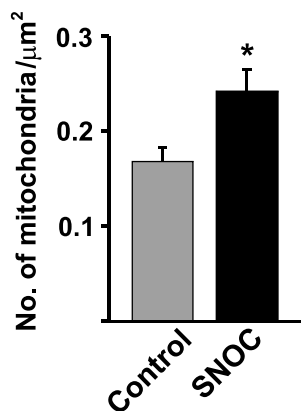

\section{g}

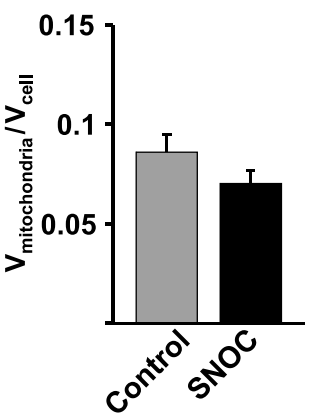

SNOC

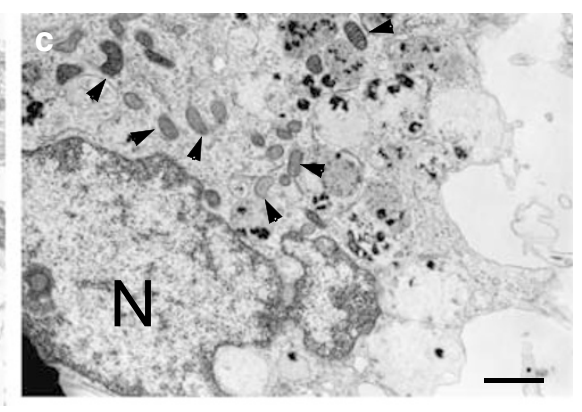

SNOC

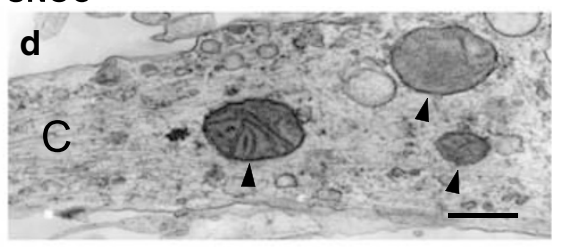

e

SNOC

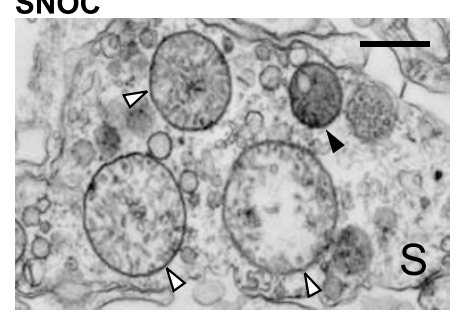

Figure 2 Mitochondrial ultrastructure after SNOC exposure. TEM of cortical neurons exposed to SNOC $(200 \mu \mathrm{M})$ at $4 \mathrm{~h}$. Cultures were pretreated with caspase inhibitor, NADFMK methyl ester $(80 \mu \mathrm{M})$, to block potential downstream caspase activation. For TEM analyses cultures were embedded in situ. (a) Electron microscopy (EM) micrograph of a thin section of a control neuron, showing the nucleus $(\mathrm{N})$ and filamentous mitochondria in the perinuclear region (arrowheads). (b) EM micrograph of a neuronal process of a control neuron. Mitochondria exhibit filamentous morphology. (c) EM micrograph of a SNOC-exposed neuron at $4 \mathrm{~h}$ showing nuclear condensation (N) and small, short mitochondria in the perinuclear region (arrowheads). (d) Micrograph of a neuronal process of a SNOC-exposed neuron at $4 \mathrm{~h}$ with globular mitochondria (black arrowheads). (e) Occasionally, mitochondrial matrix swelling is observed, characterized by light matrix, and cristae loss (white arrowhead) next to a mitochondrion with electron dense matrix (black arrowhead). The lack of cytoskeletal components in (e) labeled with $\mathrm{C}$ in (c and d) indicates more severe damage owing to SNOC-exposure; (S) labels a synapse. Scale bar, $1.5 \mu \mathrm{m}$ for $(\mathbf{a}, \mathbf{c}) ; 500 \mathrm{~nm}$ for $(\mathbf{b}-\mathbf{e})$. (f) Bar graph of number of mitochondria in control and SNOC-treated sample per $\left(\mu \mathrm{m}^{2}\right)$ measured as volume density by EM stereology ( ${ }^{\star} P<0.01$, two-tailed). (g) Bar graph of quantification of mitochondrial volume over total cell volume measured as number fraction of mitochondria by EM stereology in control and SNOC-exposed neurons

demonstrated that the number of mitochondria was $0.17 \pm 0.01 / \mu \mathrm{m}^{2}$ for the control sample and $0.24 \pm 0.02 / \mu \mathrm{m}^{2}$ for the SNOC-treated sample; the difference being statistically significant, thus indicating that mitochondrial number was increased by SNOC (Figure 2f). However, mitochondria volume per cell volume was not significantly altered (Figure $2 \mathrm{~g}$ ). The ratio of volume occupied by mitochondria over the total cellular volume in SNOC-treated neurons was $0.07 \pm 0.007$ compared to $0.08 \pm 0.009$ of the control neurons (Figure $2 \mathrm{f}$ ). Collectively, these results suggest that nitrosative stress induces mitochondrial fission in neurons accompanied by ultrastructural changes, rounded as opposed to elongated form, but without significant change to mitochondrial membrane architecture.

Mitochondrial fission and Bax foci formation. Mitochondrial fission by nitrosative stress might be part of an early neuronal response and mechanism to repair injury, thereby promoting cell survival. Alternatively, mitochondrial fission might increase the susceptibility to neuronal cell death. To explore the relationship between mitochondrial fission and cell death-related events, cortical neuronal cultures expressing DsRed2-Mito plus EGFP-Bax were exposed to $200 \mu \mathrm{M}$ SNOC. Bax, a proapoptotic member of the Bcl-2 family, is normally localized in the cytoplasm or evenly distributed on the mitochondrial outer membrane in living cells. However, Bax forms large foci on the mitochondrial outer membrane in the early phase of apoptosis in many cell types. ${ }^{21,22}$ Here, we chose Bax subcellular redistribution as a parameter to evaluate cell death-related events, because Bax is required for NO-related neuronal demise. ${ }^{9}$ Before SNOC exposure EGFP-Bax displayed cytosolic distribution in neurons (Figure $3 a$ and $a^{\prime}$ ). However, after SNOC exposure, EGFP-Bax clustered into discrete foci on mitochondria and was found either on tips of mitochondria or formed bridges between dividing mitochondria (Figure $3 b$ and $b^{\prime}$ ). Fluorescence micrographs 

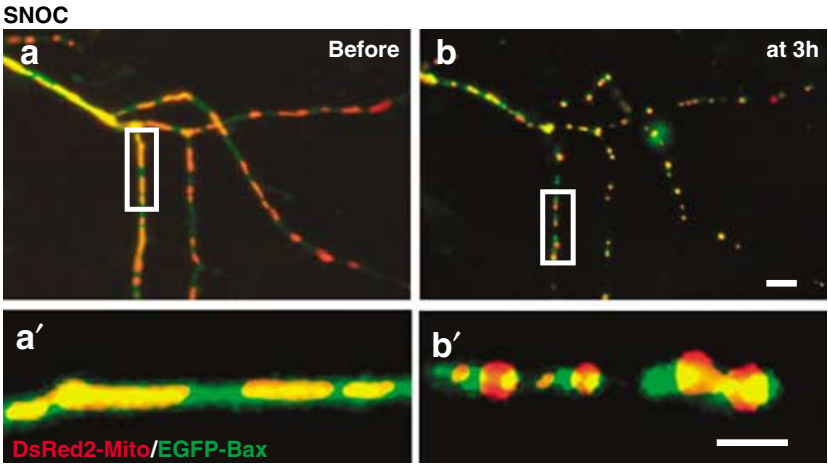

Figure 3 Mitochondrial fission and Bax foci formation on mitochondria. Neurons were co-transfected with DsRed2-Mito plus EGFP-Bax and images were taken before and after exposure to SNOC $(175 \mu \mathrm{M})$. (a) Mitochondria appear filamentous and Bax of cytoplasmic distribution in neurites before SNOC exposure. (b) At $3 \mathrm{~h}$ after SNOC exposure the EGFP-Bax pattern changed from dispersed to discrete foci on mitochondria. $\left(\mathbf{a}^{\prime}\right)$ and $\left(\mathbf{b}^{\prime}\right)$ are higher magnifications of the selected areas marked by the white rectangular in (a) and (b), respectively. Scale bar, $10 \mu \mathrm{m}$ indicated that the diameter of EGFP-Bax puncta was comparable to that of sub-resolution fluorescent beads, suggesting that Bax was confined to specific membrane areas on divided mitochondria. These results suggest that Bax might be recruited upon activation of mitochondrial fission complexes.

Bcl-xL prevents Bax foci formation and cell death without blocking mitochondrial fission. Antiapoptotic $\mathrm{Bcl}-\mathrm{xL}$ antagonizes cell death by interacting with proapoptotic Bax. ${ }^{23}$ Therefore, we tested the effects of $\mathrm{Bcl}-$ $\mathrm{xL}$ on SNOC-induced mitochondrial fission. Mitochondrial morphology in neurons transfected with DsRed2-Mito alone, in combination with Bax-EGFP, Bcl-xL, or EGFP-Bax and $\mathrm{Bcl}-\mathrm{xL}$ was assessed by fluorescence imaging. Bcl-xL plus EGFP-Bax-expressing neurons exhibited filamentous mitochondrial morphology before SNOC exposure (Figure 4a), and their average length was slightly but not significantly increased compared to EGFP-Bax only

\section{Bcl-xL/SNOC}
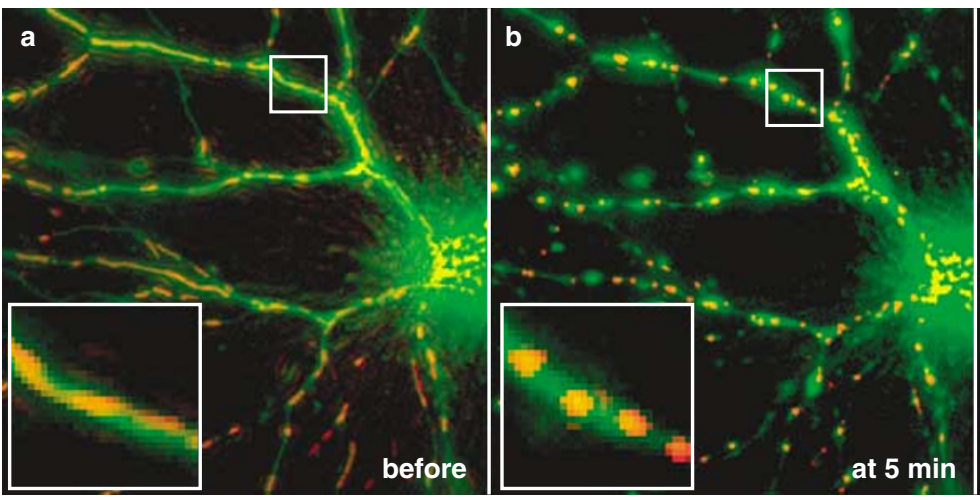

DsRed2-Mito/EGFP-Bax

d

Frag.Mitochondria at $1.5 \mathrm{hr}$

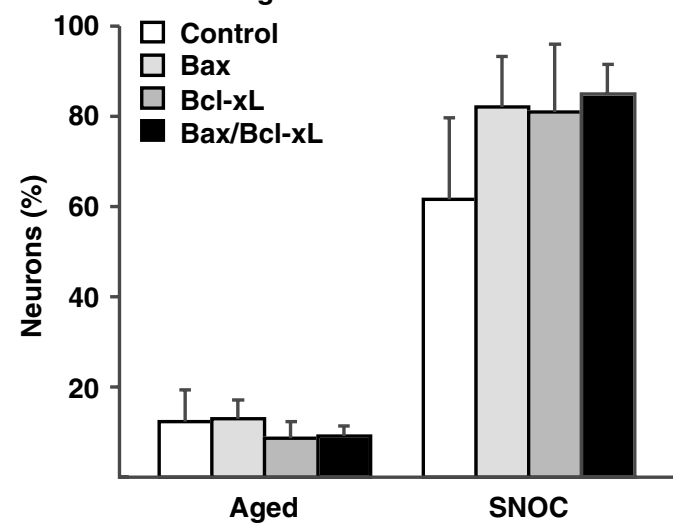

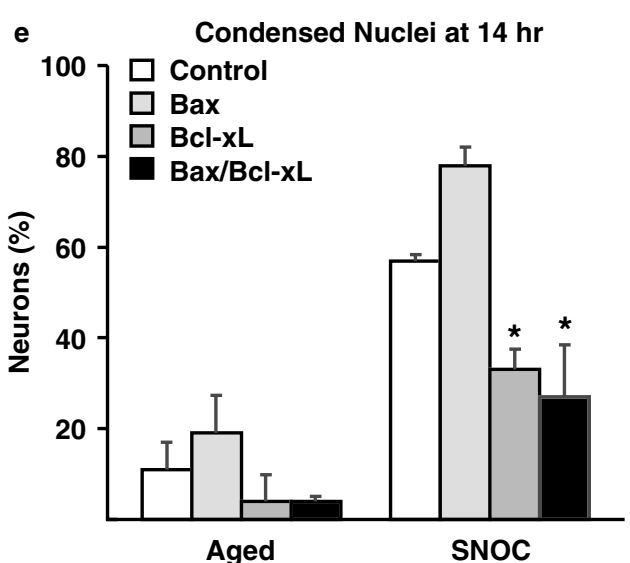

Figure $4 \mathrm{Bcl}-\mathrm{xL}$ prevents Bax translocation to mitochondria but not mitochondrial fission. Time lapse images of a neuron co-expressing DsRed2-Mito, EGFP-Bax plus pcDNA-Bcl-xL before (a), at $5 \mathrm{~min}(\mathbf{b})$, and at $4 \mathrm{~h}$ (c) after $175 \mu \mathrm{M} \mathrm{SNOC}$ exposure. Insert shows an enlargement of the area marked within the rectangular. Scale bar, $10 \mu \mathrm{m}$. (d) Percentage of mitochondrial fragmentation of neurons transfected with DsRed2-Mito alone; DsRed2-Mito plus EGFP-Bax; DsRed2-Mito plus pcDNA3-Bcl- $\mathrm{x}_{\mathrm{L}}$; or all three vectors together in response to aged or fresh SNOC $(175 \mu \mathrm{M})$ at $1.5 \mathrm{~h}$. A neuron was counted as one with fragmented mitochondria when $90 \%$ of the mitochondria had small and round morphology as shown in Figure $1 b$. Data are shown as means \pm S.E.M. from three independent experiments. (e) Percentage of cell death of neurons transfected with DsRed2-Mito; DsRed2-Mito plus EGFP-Bax; DsRed2-Mito plus Bcl-X $\mathrm{X}_{\mathrm{L}}$; or DsRed2-Mito plus EGFP-Bax, Bcl-X after exposure to aged or fresh SNOC (175 $\mu$ M). Cell death was determined after fixation of neuronal cultures at $14 \mathrm{~h}$ and by counting neurons with shrunken soma and condensed chromatin after Hoechst 33342 labeling $\left({ }^{*} P<0.05\right.$ when compared to control transfections; $n=3$ replicates as means \pm S.E.M.) 
expressing neurons $(1.45 \pm 0.1 \mu \mathrm{m} ; n=28$ and $1.2 \pm 0.06 \mu \mathrm{m}$; $n=54$, respectively). After SNOC insult mitochondria converted from long filaments to small and round organelles. Notably, EGFP-Bax remained in the cytosol, although in some neurons dendritic swellings (varicosities) started to appear, which increased in size at $4 \mathrm{~h}$ (Figure $4 \mathrm{~b}$ and $\mathrm{c}$ ). Thus although $\mathrm{Bcl}-\mathrm{xL}$ prevents the recruitment of Bax into discrete spots on the mitochondrial outer membrane, it does not alleviate the occurrence of mitochondrial fission by SNOC.

We next quantified mitochondrial fragmentation and cell death in the various transfectants. Expression of EGFP-Bax alone did not induce mitochondrial fission or cell death in cortical neurons (Figure 4d and e). Neurons expressing Bcl-xL underwent mitochondrial fission to the same degree as control neurons following SNOC insult (Figure 4d). Thus Bcl-xL does not interfere with mitochondrial fission triggered by nitrosative stress. However, when we scored cell death by counting transfected neurons with condensed nuclei after Hoechst 33342 labeling, we found that EGFP-Bax exacerbated, whereas $\mathrm{Bcl}-\mathrm{xL}$ significantly mitigated neuronal cell death by SNOC at $14 \mathrm{~h}$ (Figure 4e). Thus, SNOC-mediated mitochondrial fission appears to occur upstream or independently of the activity of $\mathrm{Bcl}-2$ family members in this experimental paradigm.

Mfn1 or Drp1 ${ }^{\text {K38A }}$ blocks mitochondrial fission and Bax translocation. Next we asked how dynamin-related fission/ fusion GTPases modulate SNOC-induced Bax aggregation on mitochondria by altering the expression levels and activity of Drp1 or Mfn1. Neurons were co-transfected with plasmids encoding DsRed2-Mito, EGFP-Bax, and either Mfn1 or dominant-negative $\operatorname{Drp1}\left(\operatorname{Drp} 1^{\mathrm{K} 38 \mathrm{~A}}\right)$. Mitochondrial fission and Bax distribution were monitored by $3 \mathrm{D}$ fluorescence microscopy. SNOC exposure resulted in profound mitochondrial fission and foci formation of EGFP-Bax on mitochondria at $4 \mathrm{~h}$ (Figure 5a). Remarkably, in neurons expressing Mfn1 or Drp1 ${ }^{\mathrm{K} 38 \mathrm{~A}}$, mitochondria retained of elongated tubular mitochondrial morphology, and EGFPBax retained cytosolic distribution upon SNOC exposure (Figure $5 b$ and $c$ ). Quantitative analysis demonstrated that Mfn1 or Drp1 ${ }^{\mathrm{K} 38 \mathrm{~A}}$ blocked SNOC-induced mitochondrial fission as well as Bax foci formation on mitochondria (Figure 4d). This further supports our previous finding that Mfn1 and Drp1 $1^{\mathrm{K} 38 \mathrm{~A}}$ partially block SNOC-induced neuronal cell death. ${ }^{17}$ These results suggest that mitochondrial fission is a prerequisite for Bax cluster formation on mitochondria and subsequent neuronal cell death upon nitrosative stress.

\section{Effects of glycolytic versus mitochondrial ATP depletion on mitochondrial morphology and Bax foci} formation. NO inhibits mitochondrial respiration and leads to a dramatic decline in the ATP/ADP ratio. ${ }^{7,17}$ Thus, it is conceivable that low cellular or mitochondrial ATP levels may represent a signal to ignite the mitochondrial fission machinery. To elucidate this question, neurons were cotransfected with plasmids encoding EGFP-Bax plus DsRed2Mito in order to monitor mitochondrial morphology and Bax foci formation in response to different ATP depleting agents. Neurons were followed by time lapse microscopy during a $4 \mathrm{~h}$ time period (Figure 6 and Table 1). To block ATP production

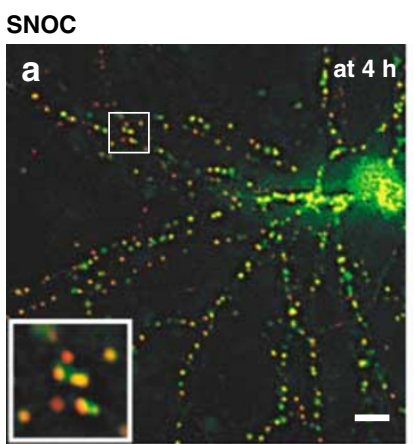

\section{Mfn1/SNOC}

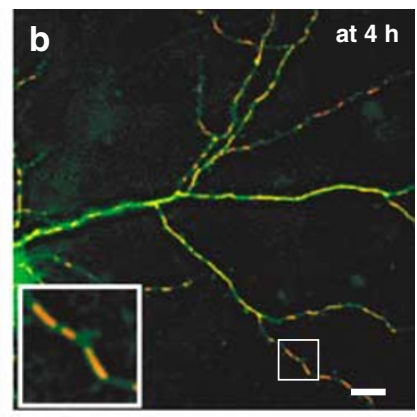

Drp1 ${ }^{\mathrm{K} 38 \mathrm{~A}} / \mathrm{SNOC}$

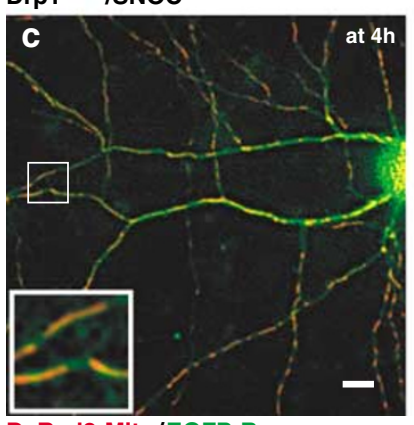

d $\quad$ Frag. Mitochondria at $4 \mathrm{~h}$

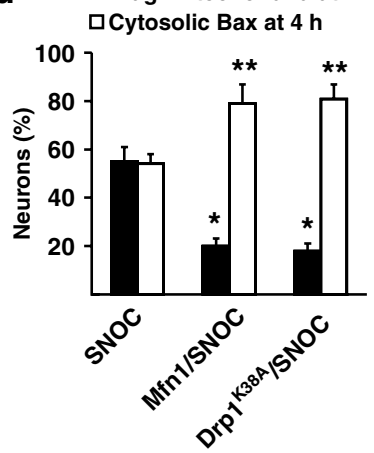

Figure $5 \mathrm{Mfn} 1$ or $\mathrm{Drp}^{\mathrm{K} 38 \mathrm{~A}}$ inhibits mitochondrial fission and Bax redistribution. Representative images of neurons co-transfected with DsRed2-Mito and EGFP-Bax (a) and additionally either pcDNA3-Mfn1 (b) or pcDNA3-Drp ${ }^{1 \mathrm{~K} 38 \mathrm{~A}}$ (c). Live neurons were imaged by fluorescence microcopy at $4 \mathrm{~h}$ after SNOC $(200 \mu \mathrm{M})$ exposure. Scale bar, $10 \mu \mathrm{m}$. (d) Quantification of the Mfn1 or Drp ${ }^{\mathrm{K} 38 \mathrm{~A}}$ effect on mitochondrial fragmentation and cytosolic localization of EGFP-Bax. $\left({ }^{\star} P<0.005\right.$ compared to neurons expressing DsRed2-Mito and EGFP-Bax, $n=3$ replicates) as well as Bax foci formation on mitochondria ( ${ }^{\star *} P<0.01, n=3$ replicates). Data are representative of three independent experiments

via glycolytic pathways, glucose was replaced with 2deoxyglucose (2DG; $10 \mathrm{mM}$; Figure $6 \mathrm{~b}$ ). 2DG is a glucose analog, which is phosphorylated by hexokinase, but is not further metabolized. ${ }^{24}$ In addition, we tested the effects of iodoacetate $(1 \mathrm{mM})$, an irreversible inhibitor of glyceraldehyde-3-phosphate dehydrogenase (GAPDH; Figure 6c). ${ }^{25}$ Pyruvate $(1 \mathrm{mM})$ was added in these experiments as a mitochondrial substrate to allow oxidative phosphorylation. Furthermore, to mimic ATP depletion by stress signalling, 5-aminoimidazole-4-carboxamide ribonucleoside (AICAR), an activator of AMP-activated protein kinase, was tested. ${ }^{26}$ Mitochondrial ATP production was blocked by the ATP synthase inhibitor oligomycin $(2 \mu \mathrm{g} / \mathrm{ml}$; Figure $6 \mathrm{~d})$, by the uncoupler carbonyl cyanide 4-(trifluoromethoxy)phenylhydrazone (FCCP, $1 \mu \mathrm{M})$, or by a combination of both (Figure 6e). For comparison, effects of SNOC $(100 \mu \mathrm{M})$ treatment were also included in Table 1; however, these data were from an independent set of experiments (Figure 6a). To eliminate potential secondary effects owing to plasma membrane depolarization elicited by low ATP levels, we blocked $N$-methyl-D-aspartate and alphaamino-3-hydroxy-5-methyl-4-isoxazolepropionate receptors and voltage-gated $\mathrm{Ca}^{2+}$ channels (except for the SNOC condition) with MK-801 $(10 \mu \mathrm{M})$, NBQX $(20 \mu \mathrm{M})$, and nifedipine $(1 \mu \mathrm{M})$, respectively. 


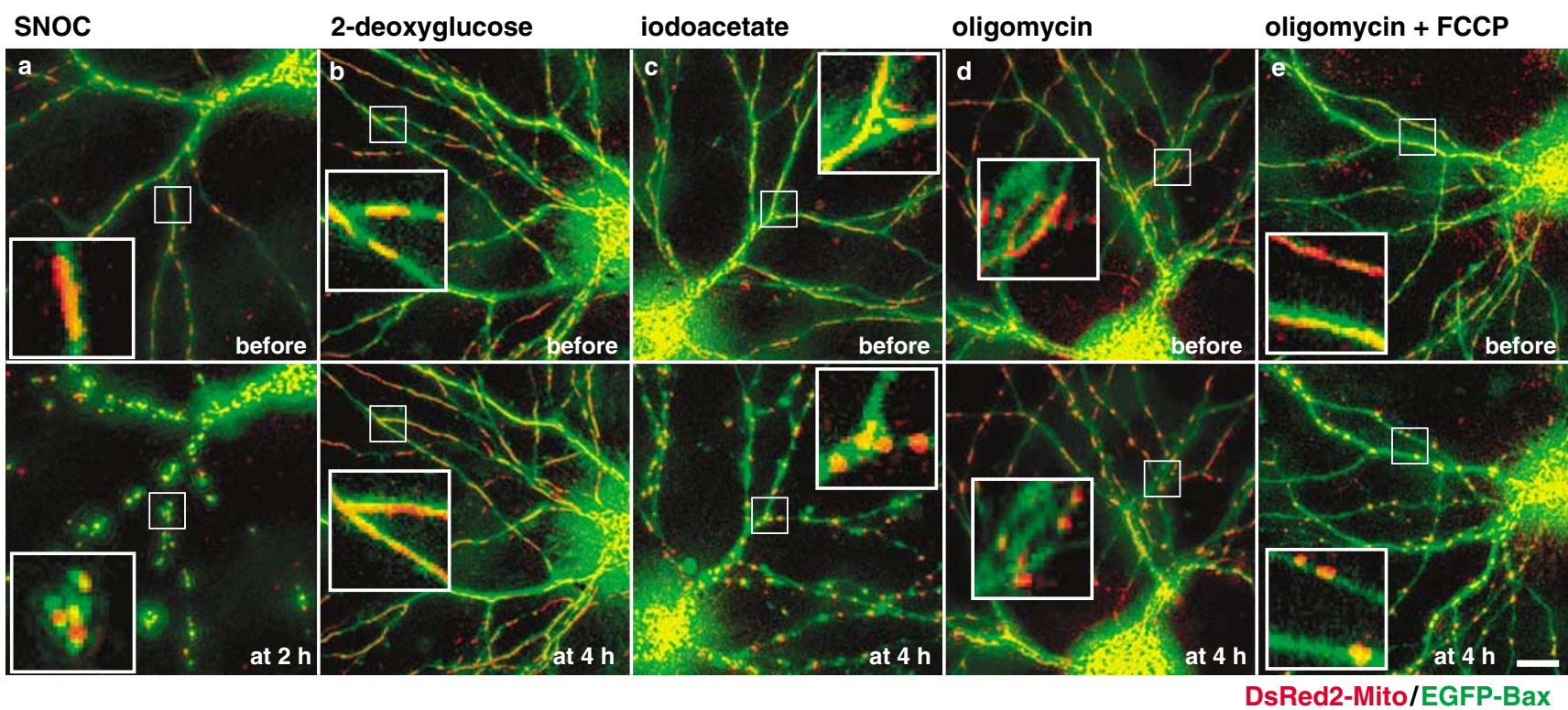

Figure 6 Effects of glycolytic and mitochondrial inhibitors on mitochondrial morphology and Bax foci formation. Representative dehazed projection images of time lapse recordings of cortical neurons expressing DsRed2-Mito and EGFP-Bax. The top row shows a neuron before and the bottom row after exposure to various agents at 2 or $4 \mathrm{~h}$. Neuron exposed to SNOC $(75 \mu \mathrm{M})(\mathbf{a})$, to $2 \mathrm{DG}(10 \mathrm{mM})$ without glucose, but in the presence of pyruvate $(1 \mathrm{mM})(\mathbf{b})$, to iodoacetate $(1 \mathrm{mM})$ in the presence of glucose $(25 \mathrm{mM})$ plus pyruvate $(1 \mathrm{mM})(\mathbf{c})$, to oligomycin $(2 \mu \mathrm{g} / \mathrm{ml})(\mathbf{d})$, or to oligomycin $(2 \mu \mathrm{g} / \mathrm{ml})$ plus FCCP $(1 \mu \mathrm{M})(\mathbf{e})$. Treatment and imaging were carried out in HBSS containing MK801 $(10 \mu \mathrm{M}), \mathrm{NBQX}(20 \mu \mathrm{M})$, and nifedipine $(1 \mu \mathrm{M})$, except for SNOC, which was carried out in Hibernate medium. Insert shows a zoom of the area marked by the white used rectangle. Scale bar, $10 \mu \mathrm{m}$

Using time lapse microscopy and computational analysis we were able to distinguish abrupt from gradual mitochondrial length shortening (defined in Materials and Methods). In addition, we measured mitochondrial numbers. This allowed us to distinguish between mitochondrial shortening owing to fission or only a simple shape change by temporal crosscorrelation. A negative coefficient (close to -1 ) is obtained when a decrease in mitochondrial length occurs with an increase in number, signifying mitochondrial fission. Conversely, when only the mitochondrial length changes, without change in number, the coefficient is closer to zero, indicating that no fission occurred.

Considering only change in mitochondrial length, but not the presence or absence of fission, SNOC or iodoacetate treatment induced abrupt mitochondrial shortening above sporadic background (Table 1). Oligomycin plus FCCPtreated neurons had a trend towards an increased incidence of abrupt mitochondrial shortening. Bax foci formation was characteristic for SNOC-triggered and sporadic abrupt mitochondrial shortening, but was not typical for iodoacetate- and oligomycin plus FCCP-induced mitochondrial shortening. Bax foci formation without mitochondrial shortening was never observed. The mitochondrial length averaged over all analyzed neurons was significantly shorter for iodoacetate, FCCP, and oligomycin plus FCCP-exposed neurons. Notably, mitochondria became significantly longer in 2DG and AICAR exposed neurons, and Bax foci formation was not observed.

Mitochondrial fission was common during SNOC-triggered mitochondrial shortening, illustrated by a negative crosscorrelation coefficient (Table 1). In contrast, there was significantly less correlation for iodoacetate and oligomycin plus FCCP treatments, shown by a coefficient closer to zero.
A similar trend was also observed for FCCP treatment. This indicates that under these conditions mitochondrial shortening is due to a shape change instead of mitochondrial fission. This conclusion is also supported by our images (Figure 6). Fissioned or fragmented mitochondria appear as tightly packed puncti, characteristic for SNOC-exposed neurons (Figure 6a). By contrast, mitochondria which only underwent a morphology change appear more sparse, larger, and beaded (Figure 6c-e).

Taken together, these data suggest that ATP depletion, as occurs during NO exposure owing to inhibition of cytochrome oxidase, ${ }^{6}$ is insufficient to signal activation of mitochondrial fission complexes and Bax aggregate formation on mitochondria.

\section{Discussion}

Here, we show that $\mathrm{NO}$ donors trigger dramatic mitochondrial fission in cortical neurons. Mitochondrial fission induced by SNOC was accompanied by Bax foci formation on mitochondria at sites of division. The SNOC-induced mitochondrial fission and Bax foci formation were not mimicked by bioenergetic blockade. The antiapoptotic $\mathrm{Bcl}-\mathrm{xL}$ failed to prevent SNOC-induced changes in mitochondrial morphology, although it prevented Bax clustering on mitochondria and neuronal cell death. Moreover, Mfn1 or Drp1 ${ }^{\mathrm{K} 38 \mathrm{~A}}$ inhibited mitochondrial fission, Bax foci formation, and mitigated neuronal cell death induced by SNOC (Figure 7). Collectively, these data indicate that activation of mitochondrial fission machinery occurs upstream of the activity of Bcl-2 family members and neuronal cell death triggered by SNOC in primary cortical cultures. 
Table 1 Quantitative analyses of the effects of glycolytic and mitochondrial inhibitors on mitochondrial morphology and Bax foci formation

\begin{tabular}{|c|c|c|c|c|c|c|c|c|}
\hline & SNOC & Control & 2DG & lodoacetate & AICAR & Oligomycin & FCCP & Oligo+FCCP \\
\hline Number of neurons & 30 & 36 & 32 & 37 & 31 & 26 & 19 & 20 \\
\hline $\begin{array}{l}\text { Number of neurons with abrupt } \\
\text { shortening of mitochondria }\end{array}$ & $22^{\star \star \star}(73 \%)$ & $6(17 \%)$ & 7 (22\%) & $31^{\star \star *}(84 \%)$ & $6(19 \%)$ & $3(12 \%)$ & $2(11 \%)$ & $8(40 \%)$ \\
\hline $\begin{array}{l}\text { Number of neurons with abrupt } \\
\text { shortening of mitochondria and } \\
\text { Bax foci formation }\end{array}$ & $13(59 \%)$ & $6(100 \%)$ & $7(100 \%)$ & $2^{\star \star \star}(6 \%)$ & $6(100 \%)$ & $3(100 \%)$ & $0(0 \%)$ & $3^{*}(38 \%)$ \\
\hline $\begin{array}{l}\text { Length change of all } \\
\text { mitochondria }\end{array}$ & ND & $134 \pm 13 \%$ & $153 \pm 12 \%$ & $61 \pm 5 \% * *$ & $153 \pm 12 \%$ & $113 \pm 6 \%$ & $78 \pm 7 \%$ ** & $59 \pm 7 \%$ ** \\
\hline $\begin{array}{l}\text { Length change of mitochondria } \\
\text { in neurons without abrupt } \\
\text { mitochondrial shortening }\end{array}$ & ND & $147 \pm 14 \%$ & $182 \pm 8 \%$ ** & ND & $177 \pm 9 \% *$ & ND & ND & ND \\
\hline $\begin{array}{l}\text { Cross-correlation between } \\
\text { mitochondrial length and } \\
\text { number }\end{array}$ & $-0.69 \pm 0.04$ & $-0.62 \pm 0.6$ & $-0.55 \pm 0.8$ & $-0.2 \pm 0.08^{\star \star \star}$ & $-0.66 \pm 0.07$ & $-0.59 \pm 0.07$ & $-0.38 \pm 0.08$ & $-0.28 \pm 0.11^{*}$ \\
\hline
\end{tabular}

Abrupt shortening of mitochondria was determined after calculation of the mean skeletal length of mitochondria for each frame of DsRed2-mito time lapse recordings, based on the magnitude of first temporal derivatives of the length data. ${ }^{* \star}$ Significance at $P<0.001$ by Fisher's exact test (two-way, performed on the pooled data set) compared to the untreated control. Bax foci formation was determined from the appearance of fine punctate pattern in EGFP-Bax images. $*, \star \star \star$ Significance at $P<0.05$, respectively by Fisher's exact test compared to untreated control. Mitochondrial length (mean skeletal length) data was compared by Kruskal-Wallis statistics with Dunn's post-hoc testing comparing to the untreated control ( ${ }^{*, * *}$ significance at $P<0.05$ and 0.01 , respectively; ND, not determined). Temporal crosscorrelation between mitochondrial length and number was compared using ANOVA with Dunett's post-hoc testing compared to the untreated control. Neurons were pooled from three to four independent experiments

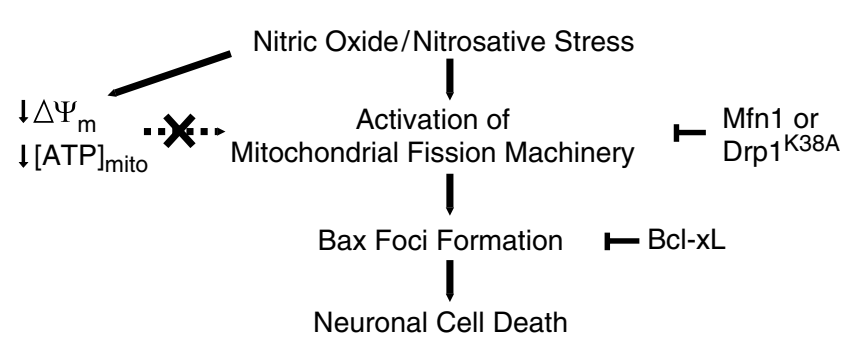

Figure 7 Model of NO-induced neuronal cell death. Mitochondrial fission induced by nitrosative stress is an early event and is followed by Bax foci formation on mitochondria. Antiapoptotic Bcl-xL functions downstream of SNOC-induced changes in mitochondrial morphology, and prevents Bax clustering on mitochondria and neuronal cell death. Moreover, Mfn1, or Drp $1^{\mathrm{K} 38 \mathrm{~A}}$ inhibits mitochondrial fission, Bax foci formation, and neuronal cell death in response to SNOC. Bioenergetic blockade does not lead to mitochondrial fission and Bax foci formation in neurons. Activation of mitochondrial fission machinery occurs upstream of the activity of Bcl-2 family members and neuronal cell death triggered by nitrosative stress in cortical neuronal cultures

How does NO evoke mitochondrial fission?. One possibility is that NO activates guanylate cyclase (GC), which in turn forms cyclic guanosine $3^{\prime}, 5^{\prime}$-monophosphate, a second messenger. Notably, NO-mediated mitochondrial biogenesis in brown fat tissue can be prevented by ODQ, a GC inhibitor. ${ }^{1}$ However, ODQ in our primary neuronal cultures failed to block fission (data not shown). Consistent with this is the finding that ODQ does not decrease excitotoxic effects of NO. ${ }^{27}$ Thus, SNOC-induced mitochondrial fission in neurons appears to be independent of the GC pathway.

Mitochondrial fusion is an energy dependent process and requires an intact inner membrane potential. ${ }^{14}$ Inhibition of mitochondrial fusion can tilt the balance toward fission. Therefore, another possibility by which NO might exert its effects on mitochondrial fission is by inhibiting mitochondrial respiration, evoking a drop in both $\Delta \Psi_{\mathrm{m}}$, and in the ATP/ADP ratio. ${ }^{5,7,17}$ Remarkably, upon discharging $\Delta \Psi_{\mathrm{m}}$ or blocking mitochondrial ATP production we found shortening of mitochondria owing to shape change, but not owing to mitochondrial fission. Moreover, this mitochondrial shortening did not lead to Bax foci formation.

In this study, we showed that NO donors, which release NO gas or are capable of direct nitrosation of other thiols trigger mitochondrial fission. Compounds like NAC, GSH, or $\mathrm{Hb}$ (oxidized) which are able to scavenge NO by nitrosation, reduce effectively mitochondrial fission events by SNOC. Whether the activity of Drp1 or other components of the fission/fusion are directly regulated by nitrosation or through other signaling molecules requires further investigation.

How does mitochondrial fission lead to neurodegeneration?. Mitochondria are in constant flux, undergoing fusion and fission. This is thought to allow effective mixing of metabolites and mtDNA, thereby ensuring full bioenergetic functionality. Furthermore, bioenergetically highly active mitochondria are associated with the filamentous phenotype. ${ }^{28,29}$ In support of this notion, we show here that by blockage of glycolysis using 2DG or triggering low energy level signaling via AICAR, neuronal mitochondria increased in length. However, it is intriguing why inhibition of GAPDH via iodoacetate has an opposite effect on mitochondrial morphology than 2DG. Further investigation is needed to elucidate this problem.

Neurons have intense energy demands. Low ATP levels may hamper axonal and dendritic transport, synaptic transmission, and pump and channel activity. Cells lacking mitochondrial fusion owing to loss of Mfn1, Mfn2, or OPA1 
reveal reduced $\Delta \Psi_{\mathrm{m}}$ and respiration. Additionally, OPA1 loss leads to abnormal cristae structure. ${ }^{15,30}$ Thus a loss of mitochondrial fusion may constitute a breach on bioenergetic functionality.

There is strong support by human genetics that a decrease in mitochondrial fusion factors cause neurodegeneration: hereditary loss-of-function mutations in OPA1 and Mfn2 result in progressive neurodegenerative disorders such as autosomal dominant optic atrophy (ADOA) and charcot-marictooth-type2A (CMT-2A), respectively. ${ }^{13}$ Furthermore, inhibition of mitochondrial fusion seems to sensitize to cell death. ${ }^{30,31}$ Here we show that nitrosative stress, implicated in acute and chronic neurodegenerative disorders, evokes extensive mitochondrial fission. Continuous mitochondrial fission might explain the mitochondrial dysfunction, known to accompany many neurodegenerative disorders.

What is the role of mitochondrial fission in the execution program of cell death?. Recent evidence reveals that components of the mitochondrial fission machinery engage with key mediators of programmed cell death. Intriguingly, Bax was found to colocalize with Drp1 and Mfn at fission sites in apoptotic cells. ${ }^{32}$ Thus, it has been postulated that Bax may participate directly in apoptotic mitochondrial fission.

We show that Drp $1^{\mathrm{K} 38 \mathrm{~A}}$ and Mfn1 block both mitochondrial fission as well as Bax foci formation on mitochondria. This suggests that the assembly of mitochondrial fission complexes occurs upstream of Bax foci formation. We speculate that assembled fission complexes may facilitate Bax activation and insertion into the mitochondrial outer membrane. Our results are in agreement with Drp1 knockdown studies in cancer cell lines, which exhibit reduced Bax accumulation on mitochondria following etoposide exposure. ${ }^{33}$ Furthermore, knockdown of Fis1 blocks Bax translocation to mitochondria. ${ }^{18}$ However, in some studies Drp $1^{\mathrm{K} 38 \mathrm{~A}}$ or Drp1 knockdown had no effect on Bax dynamics. ${ }^{12,18}$ A possible explanation might be that Drp $1^{\mathrm{K} 38 \mathrm{~A}}$ or Drp1 knockdown in these scenarios was less effective than in primary neurons. Alternatively, this might be due to differences in cell types or stimuli.

$\mathrm{Bcl}-\mathrm{xL}$ in our study did not prevent the SNOC-induced mitochondrial fission despite the fact that it blocked Bax foci formation and lessened neuronal cell death. This finding agrees with previous reports that $\mathrm{Bcl}-\mathrm{xL}$ blocks cytochrome $c$ release and cell death, but fails to inhibit mitochondrial fission in Fis1 overexpressing cells. ${ }^{34}$ This further strengthens the idea that Bcl-2 family members may act downstream of mitochondrial fission.

Translocation, foci formation, and activation Bax on the outer mitochondrial membrane leads to permeabilization of the outer mitochondrial membrane, and release of proapoptotic factors including cytochrome $c$, and AIF in a wide variety of cell types. ${ }^{35}$ We recently reported that neurons die by caspase-independent cell death, and AIF as well as cytochrome $c$ remain located in mitochondria. ${ }^{17}$ Thus an intriguing question emerges whether Bax has other functions besides permeabilization of the outer mitochondrial membrane in the SNOC-induced neuronal cell death. Bax foci may recruit not only Drp1 but Mfn2, preventing further fusion which could be detrimental for cells. ${ }^{32}$ Alternatively binding of Bax to the mitochondrial permeability transition pore complex and its activation by interaction with the adenine nucleotide translocase has been shown in mammalian mitochondria. ${ }^{36}$ This could cause an outer membrane permeabilization-independent deleterious effect on neuronal mitochondria leading to neuronal demise. ${ }^{37}$

In summary, components of the mitochondrial fission/fusion machinery may represent new drug targets to antagonize the progression of common neurodegenerative disorders in which nitrosative stress plays a role.

\section{Materials and Methods}

Plasmids and reagents. The pDsRed2-Mito vector was purchased from Clontech. pEGFP-Bax, and Drp1 ${ }^{\mathrm{K} 38 \mathrm{~A}}$, and pcDNA3-Mfn1 vectors were gifts from Dr. RJ Youle (NINDS), Dr. A.M. van der Bliek (UCLA), and Dr. MT Fuller (Stanford University), respectively. All plasmids were purified using the endotoxin-free plasmid preparation kit (Qiagen). Oligomycin and FCCP were dissolved in ethanol, NBQX in DMSO (all from Sigma). Sodium nitrite, 2-DG, iodoacetate, and poly-L-lysine were from Sigma. Spermine NONOate, SNAP, Hb, and L-Cysteine were purchased from Calbiochem. SNOC was freshly prepared as described previously. ${ }^{19}$

Primary cerebrocortical neuron culture and transfection. Cortical neurons were prepared from E17 Sprague-Dawley rat embryos. Cerebral cortices were isolated and incubated for $105 \mathrm{~min}$ at $37^{\circ} \mathrm{C}$ in $0.05 \%$ Trypsin in HEPESbuffered salt solution (HBSS) (Life Scientific). Cells were plated onto $12 \mathrm{~mm}$ glass coverslips (Fisher Scientific) in $1 \mathrm{ml}$ or $35 \mathrm{~mm}$ glass bottom dishes (MatTek) in $2 \mathrm{ml}$ at a density of $4.3 \times 10^{5}$ or $5.8 \times 10^{5} \mathrm{cells} / \mathrm{ml}$, respectively. All dishes were precoated prior to cell seeding with $1 \mathrm{mg} / \mathrm{ml}$ poly-L-lysine (Sigma). Neurons were grown in DMEM (HyClone) medium supplemented with $10 \%$ fetal bovine serum (Tissue Culture Biologicals), $25 \mathrm{mM}$ HEPES (pH 7.4), $2 \mathrm{mM}$ glutamine, and $25 \mathrm{U} / \mathrm{ml}$ penicillin and $25 \mathrm{mg} / \mathrm{ml}$ streptomycin (Invitrogen Corporation) and incubated at $37^{\circ} \mathrm{C}, 5 \% \mathrm{CO}_{2}$. After 5 days, half of the medium was replaced with fresh culture medium. Transfection was performed using Lipofectamine 2000 (Invitrogen Corporation) at 12-14 days in vitro. Experiments were carried out 2-4 days after transfection.

TEM and analysis. Neuronal cultures were grown on $35 \mathrm{~mm}$ glass bottom culture dishes (MatTek). After SNOC exposure, cultures were fixed with a $37^{\circ} \mathrm{C}$ solution of $2 \%$ paraformaldehyde, $2.5 \%$ glutaraldehyde in $0.1 \mathrm{M}$ sodium cacodylate (pH 7.4), and transferred to room temperature for $5 \mathrm{~min}$, and then incubated for an additional $30 \mathrm{~min}$ on ice. Fixed cultures were then rinsed three times for $3 \mathrm{~min}$ each with $0.1 \mathrm{M}$ sodium cacodylate plus $3 \mathrm{mM}$ calcium chloride $(\mathrm{pH} 7.4)$ on ice and then post-fixed with $1 \%$ osmium tetroxide, $0.8 \%$ potassium ferrocyanide (Sigma), $3 \mathrm{mM}$ calcium chloride in $0.1 \mathrm{M}$ sodium cacodylate $(\mathrm{pH} 7.4)$ for $60 \mathrm{~min}$ and then washed three times for $3 \mathrm{~min}$ with ice-cold distilled water. Cultures were finally stained overnight with $2 \%$ uranyl acetate at $4{ }^{\circ} \mathrm{C}$, dehydrated in graded ethanol baths, and embedded in Durcupan resin (Fluka). Ultrathin $(70 \mathrm{~nm})$ sections were post-stained with uranyl acetate and lead salts and evaluated by a JEOL 1200FX TEM. All reagents were purchased from TED PELLA Inc., unless otherwise indicated.

Random sampling of thin sections produced 25 SNOC images and seven control images. Images were recorded on film at 5000 magnification on a JEOL1200FX electron microscope operated at $80 \mathrm{kV}$. Stereological observations were performed as previously described ${ }^{38}$ on sections estimated to be of $70 \mathrm{~nm}$ thickness as follows. Point counting was used to determine the mitochondrial volume densities ${ }^{39}$ by overlaying a 100-point square grid on each digitized image $(2689 \times 4006$ pixels with a pixel size of $3 \mathrm{~nm}$ ) in Adobe Photoshop. Observations were made at the points of intersection ( $10 \times 10$ points $)$ of the grid. First, the volume available for mitochondria to occupy was counted on the $10 \times 10$ grid overlay of each image; by definition, the maximum count was 100 . This was accomplished by excluding those points lying on top of any nucleus present and any empty space, that is, space not occupied by cells, giving a count somewhat less than 100 . Next, the points lying directly on top of mitochondria were counted. The volume density of mitochondria was calculated by dividing the number of mitochondrial points by the available volume points. The number fraction of mitochondria was determined by counting the number of mitochondria (not points) found within the square grid and this number was divided by the area occupied by the cytoplasm in each image. Measurements on 114 control and 467 SNOC mitochondria contributed to Figure 2. 
Fluorescence microscopy and image analysis. Live cell imaging experiments were conducted in HBSS containing (in $\mathrm{mM}$ ): $140 \mathrm{NaCl}, 3.9 \mathrm{KCl}, 1.8$ $\mathrm{CaCl}_{2}, 1 \mathrm{MgCl}_{2}, 0.4 \mathrm{NaH}_{2} \mathrm{PO}_{4}, 1.2 \mathrm{Na}_{2} \mathrm{SO}_{4}, 20 \mathrm{HEPES}$, and 25 glucose at $\mathrm{pH} 7.4$ or in Hibernate- $\mathrm{E}_{\mathrm{LF}}$ medium (BrainBits Inc.) at $37^{\circ} \mathrm{C}$. Fluorescence image acquisition was performed using a Zeiss Axiovert $100 \mathrm{M}$ fluorescence microscope (Carl Zeiss; Plan Apochromat $100 \times 1.4 \mathrm{NA}$ oil for $3 \mathrm{D}$ reconstructions, Plan Apochromat $63 \times 1.4$ NA oil for time lapses or Plan-Neofluar $40 \times 0.75$ NA dry objectives for revisiting end point acquisitions), and a DG-4/Lamda10-2 combo Xe-arc illumination unit (Sutter), Lambda 10-2 emission filter wheel (Sutter), a Sensicam QE, cooled CCD camera (PCO AG, Germany) controlled by Slidebook software (Intelligent Imaging Innovations Inc.). Fluorescent proteins were excited through excitation filters for $\operatorname{EGFP}(S 490 / 20 \times)$ and DsRed2 $(\mathrm{S} 555 / 28 \times)$. Fluorescent light was collected via a triple band beamsplitter and emission filters for EGFP (S528/38 m) and for DsRed2 $(\mathrm{S} 617 / 73 \mathrm{~m})$. All filters were from Chroma. Time lapses were collected by Metamorph 7.01 software with the Multi Dimensional Acquisition feature (Molecular Devices). Z-stacks of $512 \times 512 \times 12$ bit frames $\left(0.2 \times 0.2 \times 1.5 \mu \mathrm{m}^{3}\right.$ voxel size; at $2 \times 2$ binning; $13-15$ planes $)$ were acquired at time intervals of $15 \mathrm{~min}$. The $3 \mathrm{D}$ reconstruction was performed using Volocity software (Improvision). Z-stacks overview of DsRed2-Mito/EGFP-Bax colocalization in whole neurons were dehazed by applying a highpass spatial filter designed on an empirical basis ${ }^{40}$ and $z$-projected using a custom version of Metafluor Analyst (Universal Imaging Corp.). Images were plotted at a gamma value of 1.2-1.5 to visualize dimmer details.

To analyze changes of mitochondrial length and number of time lapse experiments, image stacks were processed by custom algorithms written in Mathematica 5.2 (Wolfram Research) and Delphi 6.0 (Borland). The dehazed projection images were thresholded and skeletonized, followed by determination of skeletal length and the number of skeletons for each frame of the time lapse series. ${ }^{17}$ Temporal correlation coefficients were calculated by Fourier transformation of the first temporal derivatives of mitochondrial length and number traces and multiplication of one transform with the complex conjugate of the other, followed by inverse transformation.

To score mitochondrial fragmentation manually, neuronal cultures were fixed using $0.5 \%$ glutaraldehyde in PBS at $4^{\circ} \mathrm{C}$ for $20 \mathrm{~min}$ to preserve mitochondrial morphology and treated with $1 \%$ sodium borohydride (Aldrich) in PBS for 30 min to omit autofluorescence. Neurons expressing DsRed2-Mito with filamentous mitochondria in neurites were scored as ones without mitochondrial fragmentation whereas neurons showing $90 \%$ of the small and round mitochondria were scored as neurons with fragmented mitochondria.

To assess neuronal cell death, cultures on glass coverslips were exposed to SNOC in Hibernate- $E_{L F}$ medium. After $14 \mathrm{~h}$ incubation, cultures were fixed as described above. Nuclear morphology was evaluated using Hoechst $33342(1 \mu \mathrm{g} /$ $\mathrm{ml})$. DsRed2-Mito-expressing neurons were scored as dead when their soma was shrunken and their nuclei were condensed. Three coverslips were used for each treatment. All quantitative experiments were carried at least three times with separate neuronal preparations.

Statistical analysis. Data from populations of mitochondria or neurons are represented as mean \pm S.E.M. Comparisons between two groups were made using a Student's $t$-test, unless otherwise stated. Statistical analyses were performed using Microsoft Excel and Graphpad Prism.

Acknowledgements. We thank Dr. RJ Youle (NINDS) for his pEGFP-Bax construct and for critical comments on the manuscript. We are also grateful to Drs. AM van der Bliek (UCLA) and MT Fuller (Stanford University), for Drp1K38A, and pcDNA3-Mfn1 vectors, respectively. We thank Dr. B Bossy, and Ms. N Sejbuk for the preparation of neuronal cultures as well as Drs. MJ Barsoum, and N Brady for their input on an earlier version of this manuscript. This work was supported by $\mathrm{NIH}$ Grants: R01 NS44314, R01 NS047456, R01 EY016164, P01 Al055789, and The American Parkinson Disease Association Inc. (to E B-W); NIH Grants: P41RR04050, R01 NS14718 (to MHE); NIH Grants: R01 EY05477, R01 EY09024, R01 NS043242, R01 NS044326, R01 NS046994, R01 NS047973, P01 Al05578 and P01 HD29587 (to SAL).

1. Nisoli E, Clementi E, Paolucci C, Cozzi V, Tonello C, Sciorati C et al. Mitochondrial biogenesis in mammals: the role of endogenous nitric oxide. Science 2003; 299: 896-899.

2. Dawson VL, Dawson TM. TM nitric oxide in neurodegeneration. Prog Brain Res 1998; 118 : 215-229.
3. Bonfoco E, Krainc D, Ankarcrona M, Nicotera P, Lipton SA. Apoptosis and necrosis: two distinct events induced, respectively, by mild and intense insults with $\mathrm{N}$-methyl-D-aspartate or nitric oxide/superoxide in cortical cell cultures. Proc Natl Acad Sci USA 1995; 92 : 7162-7166.

4. Duncan AJ, Heales SJ. Nitric oxide and neurological disorders. Mol Aspects Med 2005; 26: 67-96.

5. Radi R, Rodriguez M, Castro L, Telleri R. Inhibition of mitochondrial electron transport by peroxynitrite. Arch Biochem Biophys 1994; 308: 89-95.

6. Brown GC, Cooper CE. Nanomolar concentrations of nitric oxide reversibly inhibit synaptosomal respiration by competing with oxygen at cytochrome oxidase. FEBS Lett 1994; 356: 295-298.

7. Brorson JR, Schumacker PT, Zhang H. Nitric oxide acutely inhibits neuronal energy production. The Committees on Neurobiology and Cell Physiology. J Neurosci 1999; 19: 147-158.

8. Bossy-Wetzel E, Talantova MV, Lee WD, Schölzke MN, Harrop A, Mathews E et al. Crosstalk between nitric oxide and zinc pathways to neuronal cell death involving mitochondrial dysfunction and p38-activated K+ channels. Neuron 2004; 41: 351-365.

9. Ghatan S, Larner S, Kinoshita Y, Hetman M, Patel L, Xia Z et al. p38 MAP kinase mediates bax translocation in nitric oxide-induced apoptosis in neurons. J Cell Biol 2000; 150: 335-347.

10. Bleazard W, McCaffery JM, King EJ, Bale S, Mozdy A, Tieu Q et al. The dynamin-related GTPase Dnm1 regulates mitochondrial fission in yeast. Nat Cell Biol 1999; 1: 298-304.

11. Smirnova E, Griparic L, Shurland DL, van der Bliek AM. Dynamin-related protein Drp1 is required for mitochondrial division in mammalian cells. Mol Biol Cell 2001; 12: 2245-2256.

12. Frank S, Gaume B, Bergmann-Leitner ES, Leitner WW, Robert EG, Catez F et al. The role of dynamin-related protein 1, a mediator of mitochondrial fission, in apoptosis. Dev Cell 2001; 1: 515-525.

13. Chan DC. Mitochondria: dynamic organelles in disease, aging, and development. Cell 2006; 125: 1241-1252.

14. Meeusen S, McCaffery JM, Nunnari J. Mitochondrial fusion intermediates revealed in vitro. Science 2004; 305: 1747-1752.

15. Griparic L, van der Wel NN, Orozco IJ, Peters PJ, van der Bliek AM. Loss of the intermembrane space protein Mgm1/OPA1 induces swelling and localized constrictions along the lengths of mitochondria. J Biol Chem 2004; 279: 18792-18798.

16. Chen $\mathrm{H}$, Chomyn $\mathrm{A}$, Chan DC. Disruption of fusion results in mitochondrial heterogeneity and dysfunction. J Biol Chem 2005; 280: 26185-26192.

17. Barsoum MJ, Yuan H, Gerencser AA, Liot G, Kushnareva Y, Graber S et al. Nitric oxideinduced mitochondrial fission is regulated by dynamin-related GTPases in neurons. $E M B O$ J 2006; 25: 3900-3911.

18. Lee YJ, Jeong SY, Karbowski M, Smith CL, Youle RJ. Roles of the mammalian mitochondrial fission and fusion mediators Fis1, Drp1, and Opa1 in apoptosis. Mol Biol Cell 2004; 15: 5001-5011.

19. Lei SZ, Pan ZH, Aggarwal SK, Chen HS, Hartman J, Sucher NJ et al. Effect of nitric oxide production on the redox modulatory site of the NMDA receptor-channel complex. Neuron 1992; 8: 1087-1099.

20. Cheung WS, Bhan I, Lipton SA. Nitric oxide (NO ${ }^{\bullet}$ ) stabilizes whereas nitrosonium $\left(\mathrm{NO}^{+}\right)$enhances filopodial outgrowth by rat retinal ganglion cells in vitro. Brain Res 2000; 868: $1-13$.

21. Wolter KG, Hsu YT, Smith CL, Nechushtan A, Xi XG, Youle RJ. Movement of Bax from the cytosol to mitochondria during apoptosis. J Cell Biol 1997; 139: 1281-1292.

22. Nechushtan A, Smith CL, Hsu YT, Youle RJ. Conformation of the Bax C-terminus regulates subcellular location and cell death. EMBO J 1999; 18: 2330-2341.

23. Danial NN, Korsmeyer SJ. Cell death: critical control points. Cell 2004; 116: 205-219.

24. Vergun $\mathrm{O}$, Han YY, Reynolds IJ. Glucose deprivation produces a prolonged increase in sensitivity to glutamate in cultured rat cortical neurons. Exp Neurol 2003; 183: 682-694.

25. Sabri MI, Ochs S. Inhibition of glyceraldehyde-3-phosphate dehydrogenase in mammalian nerve by iodoacetic acid. J Neurochem 1971; 18: 1509-1514.

26. Corton JM, Gillespie JG, Hawley SA, Hardie DG. 5-aminoimidazole-4-carboxamide ribonucleoside. A specific method for activating AMP-activated protein kinase in intact cells? Eur J Biochem 1995; 229: 558-565.

27. Marks JD, Boriboun C, Wang J. Mitochondrial nitric oxide mediates decreased vulnerability of hippocampal neurons from immature animals to NMDA. J Neurosci 2005; 25: $6561-6575$.

28. Ono T, Isobe K, Nakada K, Hayashi Jl. Human cells are protected from mitochondrial dysfunction by complementation of DNA products in fused mitochondria. Nat Genet 2001; 28: $272-275$

29. Skulachev VP. Mitochondrial filaments and clusters as intracellular power-transmitting cables. Trends Biochem Sci 2001; 26: 23-29.

30. Frezza C, Cipolat S, Martins de Brito O, Micaroni M, Beznoussenko GV, Rudka T et al. OPA1 controls apoptotic cristae remodeling independently from mitochondrial fusion. Cell 2006; 126: 177-189.

31. Olichon A, Baricault L, Gas N, Guillou E, Valette A, Belenguer P et al. Loss of OPA1 perturbates the mitochondrial inner membrane structure and integrity, leading to cytochrome $c$ release and apoptosis. J Biol Chem 2003; 278: 7743-7746.

32. Karbowski M, Lee YJ, Gaume B, Jeong SY, Frank S, Nechushtan A et al. Spatial and temporal association of Bax with mitochondrial fission sites, Drp1, and Mfn2 during apoptosis. J Cell Biol 2002; 159: 931-938. 
33. Neuspiel M, Zunino R, Gangaraju S, Rippstein P, McBride H. Activated mitofusin 2 signals mitochondrial fusion, interferes with Bax activation, and reduces susceptibility to radica induced depolarization. J Biol Chem 2005; 280: 25060-25070.

34. Frieden M, James D, Castelbou C, Danckaert A, Martinou JC, Demaurex N. $\mathrm{Ca}^{2+}$ homeostasis during mitochondrial fragmentation and perinuclear clustering induced by hFis1. J Biol Chem 2004; 279: 22704-22714.

35. Martinou JC, Green DR. Breaking the mitochondrial barrier. Nat Rev Mol Cell Biol 2001; 2 63-67.

36. Belzacq AS, Vieira HL, Verrier F, Vandecasteele G, Cohen I, Prevost MC et al. Bcl-2 and Bax modulate adenine nucleotide translocase activity. Cancer Res 2003; 63: 541-546.
37. Tsujimoto Y, Nakagawa T, Shimizu S. Mitochondrial membrane permeability transition and cell death. Biochim Biophys Acta 2006 [Epub ahead of print]

38. Ruiz-Lozano P, Smith SM, Perkins G, Kubalak SW, Boss GR, Sucov HM et al. Energy deprivation and a deficiency in downstream metabolic target genes during the onset of embryonic heart failure in RXRalpha-I- embryos. Development 1998; 125: 533-544.

39. Mathieu-Costello O, Ju Y, Trejo-Morales M, Cui L. Greater capillary-fiber interface per fiber mitochondrial volume in skeletal muscles of old rats. J Appl Physiol 2005; 99: 281-289.

40. Gerencser AA, Adam-Vizi V. Selective, high-resolution fluorescence imaging of mitochondrial $\mathrm{Ca}^{2+}$ concentration. Cell Calcium 2001; 30: 311-321. 